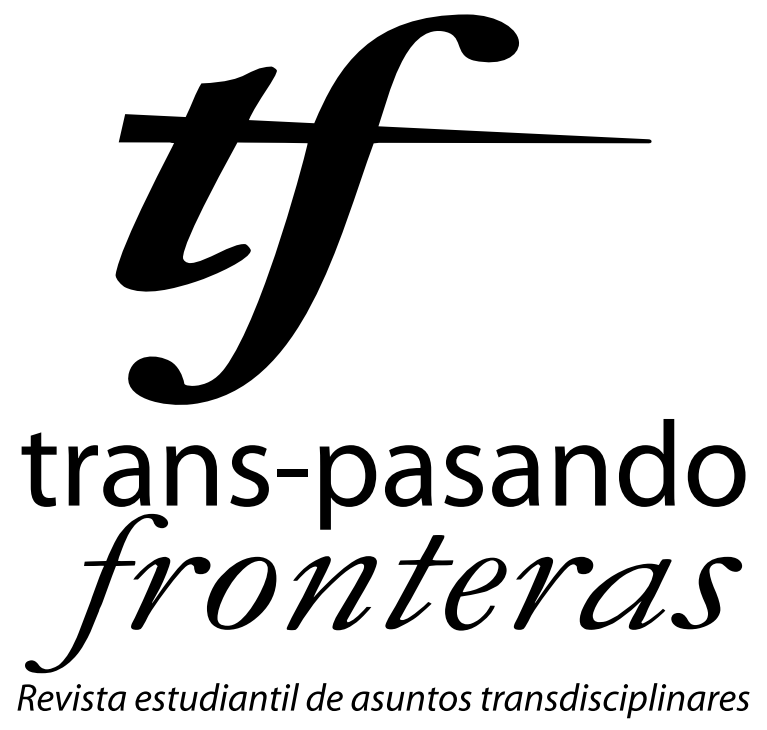

Una publicación de

Estudios

Interdisciplinarios

Jurídicos, Sociales

000 y Humanistas

ICESS

FACULTAD DE

DERECHO Y CIENCIAS

SOCIALES

然 UNIVERSIDAD 


\title{
La MANE como mecanismo legítimo de representación estudiantil
}

\author{
Natalia Muñoz ${ }^{* *}$ \\ (nati-munoz@hotmail.com)
}

\author{
Andrea Hernandez ${ }^{* * *}$ \\ (andreaht92@hotmail.com)
}

Reporte de caso recibido el 25/05/2013 y aprobado el 20/01/2013.

Como citar este artículo:

MUÑOZ, Natalia y Andrea Hernandez (2013). "La MANE como mecanismo legítimo de representación estudiantil”. En: Trans-pasando Fronteras, Núm. 3, pp. 59-69. Cali, Colombia: Centro de Estudios Interdisciplinarios, Jurídicos, Sociales y Humanistas (CIES), Facultad de Derecho y Ciencias sociales, Universidad Icesi.

\begin{abstract}
Resumen
Este articulo muestra el proceso coyuntural de la Mesa Amplia Nacional de Estudiantes, la cual surge como una forma de resistencia estudiantil, en contra de la Reforma que el gobierno Santos propone hacer a la ley 30 de 1992. Esta ley estructura la educación superior en Colombia. La MANE es un movimiento social de representación estudiantil. Ha logrado legitimidad por el uso de nuevas formas de manifestación pacífica y ganado fuerza a nivel nacional, aumentando sus posibilidades de influir en la política colombiana. Dentro de este movimiento se dieron dos momentos: el primero

* Gracias a Jennifer García e Inés Mazuera. Sin ustedes este trabajo no hubiera sido posible.

** Estudiante de Ciencia política con énfasis en Relaciones internacionales de la Universidad Icesi. Sus áreas de interés son los regímenes políticos, las instituciones políticas y los movimientos sociales. Es asistente en la investigación de "Élites y Representaciones Cali 1910-1950".

*** Estudiante de Ciencia política con énfasis en Relaciones internacionales de la Universidad Icesi. Sus áreas de interés son las políticas públicas, la diplomacia, el lobbying y los movimientos sociales. Es colaboradora en la investigación de "Redes de ciudades en el suroccidente colombiano" y se desempeña como tutora de escritura en el Centro de Recursos para el Aprendizaje (CREA) de la misma Universidad.
\end{abstract}


ligado a la resistencia estudiantil y el segundo referente a la creación de una nueva propuesta de ley para la educación.

Palabras claves:

MANE, Movimiento estudiantil, Ley 30 de 1992, Estado, Educación Superior

\section{Introducción}

El presente artículo pretende indagar cómo la Mesa Amplia Nacional Estudiantil (MANE) se convierte en una fuerza legítima de representación de estudiantes entre el 2011 y el 2012, teniendo en cuenta tres dimensiones: repertorios, demandas y dificultades. Para ello, se hace una recolección de información en: noticias, debates en el Congreso, columnas de opinión e informes especiales, además de realizar entrevistas a algunos de los actores involucrados en el movimiento. Finalmente, se hará una revisión de documentos bibliográficos que permitan abordar de manera detallada los movimientos sociales.

\section{LA MANE: nuevo mecanismo legítimo de representación}

El proyecto de Reforma a la ley 30 radicado en el Congreso por la Ministra de Educación en representación del gobierno, generó un clima de inconformidad entre los estudiantes y profesores colombianos. Por eso, los primeros decidieron reunirse en la Mesa Amplia Nacional de Estudiantes (MANE), buscando referentes unitarios de acción y organización que los convirtiera en un mecanismo legítimo de representación.

Para esto, los estudiantes no sólo utilizaron acciones tradicionales sino que crearon nuevos repertorios de movilización como los carnavales nocturnos, el pupitrazo, el abrazatón, el besatón y las caminatas hacia la capital del país, ganando así, la opinión pública y lo que nos parece aún más importante, lograron romper con las ideas preconcebidas en la población, tales como: los estudiantes son tira piedras, agresivos, vándalos y sus manifestaciones sólo causan daños y disturbios.

Sin embargo, no todo es color rosa ya que la MANE ha presentado dificultades a la hora de tomar decisiones y plantear una postura homogénea. Esto se debe a que al interior de la MANE no hay estudiantes de una sola universidad. Por el contrario, son de diferentes universidades públicas y privadas del país, que piensan distinto y perciben las cosas de forma diferente. De igual manera, existen entre ellos diferencias de tipo ideo- 
lógica y partidarias, que hacen que tengan diferentes concepciones sobre la educación y sobre los cambios que esta requiere.

\section{La MANE: ¿Un movimiento social?}

Para responder nuestra pregunta, se hace necesario mirar si la MANE es un movimiento social. Por tanto, partimos de la definición de Mauricio Archila, quien entiende a los movimientos sociales como "aquellas acciones sociales colectivas más o menos permanentes, orientadas a enfrentar injusticias, desigualdades o exclusiones, que tienden a ser propositivas en contextos históricos específicos" (Archila, 2001:18).

Entonces, teniendo en cuenta la definición mencionada anteriormente, vamos punto por punto. En primer lugar, existe una acción colectiva de los estudiantes en oposición a la acción individual del Estado. En segundo lugar, la MANE ha demostrado una permanencia en el tiempo, ya que después de haber cumplido su objetivo de no permitir la implementación de la reforma, continúa vigente con nuevos fines y dinámicas. Empero, su futuro como movimiento cohesionador sigue siendo incierto, ya que la diversidad de pensamiento e ideologías heterogéneas presentes dentro de este, puede hacer que se fragmente provocando que cada grupo tire hacia su propio lado. En tercer lugar, la MANE denota conflicto, ella enfrenta injusticias y desigualdades sociales. Según los estudiantes, la reforma agudizaría la inversión del Estado en seguridad, disminuyendo aún más su inversión en educación. En cuarto lugar, la Mesa como un movimiento contemporáneo tiende no sólo a manifestar su oposición, sino también a crear propuestas para plantear una solución. Esto se pone de manifiesto en la segunda dinámica del movimiento. No obstante, consideramos que esto podría tener implicaciones al interior de la MANE, pues podría debilitar su carácter cohesionador. Por último, respecto a la dimensión histórica, vemos que la MANE no surge de la nada, sino que más bien hace parte de un contexto internacional en el que países como República Dominicana, Puerto Rico y Chile, luchan por una educación gratuita y de calidad. Asimismo, se inscribe en un contexto neoliberal en el que la educación al igual que la salud, ya no son un derecho sino un servicio.

De igual manera, nos parece pertinente señalar las características planteadas por María Gohn (2002). Según ella, estos movimientos hacen una evaluación de la realidad social mediante redes y acciones colectivas para construir propuestas que permitan so- 
lucionar los problemas de exclusión social. Es así, como vemos que la MANE establece vínculos con padres de familia, trabajadores universitarios, profesores, representantes de los procesos de organización social y política, como el Congreso de los pueblos. En síntesis, consideramos a la MANE como un movimiento social.

\section{Y así surgió la MANE}

Según el líder de la Mesa Amplia Estudiantil Icesi Carlos Liévano, “desde el período presidencial de Álvaro Uribe ya existía un rumor de una Reforma a la ley de educación. Sin embargo, culminó el mandato de Uribe, la Reforma no se presentó y los estudiantes quedaron a la expectativa. Al subir Santos a la presidencia y durante el primer período legislativo, la reforma tampoco se llevó a cabo”. Pero, el 12 de Abril de 2011 durante el segundo período legislativo, el Ministerio de educación radicó en la Secretaria general del Senado el proyecto de Reforma a la ley 30 de 1992, principal normativa de la educación superior.

Debido a esto, los jóvenes deciden organizarse y unificar un foro permanente de discusión de los estudiantes, llamado Mesa Amplia Nacional de Estudiantes (MANE) que tiene entre sus principales objetivos hacer respetar los derechos de la población estudiantil. No obstante, esa decisión no se toma de la noche a la mañana. Desde el 2004 los estudiantes deciden reunirse con la Coordinadora Nacional Estudiantil Universitaria; para lograr que el movimiento tuviera un alcance a nivel nacional. Según Andrés Rincón, activista de la Federación Universitaria Nacional (FUN):

\footnotetext{
“tampoco podemos olvidar los intentos coordinadores vividos en 2002 y 2003, lo cual nos permite decir que por lo menos durante una década el movimiento estudiantil vivió intentos de aglutinación nacional, particularmente el proceso de la CNEU, que por varias dificultades terminó en 2006, momento a partir del cual se realizaron varios encuentros estudiantiles, de emergencia, para mirar el tema de coyuntura" (Rincón, 2012)
}

Es así como, Óscar Aponte activista de Rebeldía Estudiantil Organizada (REO) señala que se "fueron decantando elementos hasta que en el primer encuentro del ENEU [marzo de 2011] se decide que se va a crear la Mane y que se va a convocar a una sesión de concreción de lo que ésta debía ser” (Rincón, 2012). 
De igual manera, como lo manifiesta el representante de la MANE en la Universidad Icesi, Carlos Liévano, esa idea se materializa cuando los líderes de las universidades públicas invitan a una gran movilización en Bogotá el día 20 de Agosto de 2011, en la cual se da una división de las funciones, mediante tres subgrupos: mesa de articulación nacional, mesa de movilizaciones y mesa programática.

\section{Programa mínimo: las exigencias de los estudiantes}

De igual manera, se estableció un programa mínimo que constituye las demandas básicas del movimiento. Ellas giran en torno a seis ejes: Primero, educación financiada por el Estado en su totalidad; segundo, el bienestar universitario; tercero, la calidad educativa; cuarto, participación y autonomía; quinto, planeación de políticas públicas a partir de la Universidad; finalmente, libertades democráticas (apoyo a la movilización social y respeto a los derechos humanos). Más que puntos de un programa, estos temas buscan llamar la atención ya que pese a que son diversos elementos, todos giran en torno a un tema macro y concreto dentro de la mesa programática (Rincón, 2012).

En ese sentido, este programa mínimo ha sido de gran utilidad para llamar la atención y adherir estudiantes. Es decir, ha servido para llegar a universidades, facultades y carreras. Además este programa dio a entender que mientras el gobierno presentaba su majestuosa propuesta, los estudiantes venían detrás de ellos, no sólo con la respuesta ante la divulgación de la ley sino que también crearon su propio dictamen y reglas de juego planteadas por ellos mismos, siendo esta la base ante la propuesta de educación.

\section{Marchas, besos y abrazos...}

Nataly Cruz una de las representantes de la MANE en la Universidad del Valle señala que la Mesa empieza a establecer pautas para todos los estudiantes del país y es así, como se convocan varias movilizaciones y marchas para presionar al gobierno en el retiro de la reforma. Las marchas se dieron, principalmente, en los meses de septiembre y octubre. En un principio, se basaban en una lucha contra el ingreso de capital privado a las universidades públicas y posteriormente, se transforma en una lucha que busca el retiro de la Reforma en su totalidad.

Sin embargo, estas dos formas tradicionales de manifestación no fueron las únicas. Ade- 
más, se utilizaron nuevos repertorios como: el "Besatón", realizado en diferentes espacios públicos del país, el cual consistía en darse besos entre los estudiantes (El Espectador, Oct 2001). También se usó el "Abrazatón”, mecanismo de protesta que consistía en darle abrazos a las busetas, al MIO, a los policías, los guardas de tránsito y transeúntes. Es por esto que "los policías les dijeron a sus superiores que nunca en su vida habían estado tan orgullosos de ser policías, algunos incluso le pidieron directamente al general Naranjo que las fotografias de los estudiantes abrazándolos sean difundías por la dirección general en todo el mundo, como ejemplo de la calidad de nuestros estudiantes y de los uniformados"”.

Otro mecanismo de protesta fueron las "marchas con antorchas", en las que los estudiantes en lugares públicos llamaban la atención del resto de la población con consignas como "el pueblo lo dice y tiene la razón, primero es lo primero: salud y educación"; "viva la u, viva, viva la u, viva, viva la universidad, no la dejes no, no la dejes no, no la dejes privatizar" y "los estudiantes no somos terroristas, terrorista el Estado, que desaparece y asesina"2.

Por otra parte, el "Pupitrazo" consistió en que los estudiantes sacaron los pupitres de las aulas a las calles (La Vanguardia, 2001). Pero, la respuesta por parte de la fuerza pública, en especial de la ESMAD fue reprimir y agredir a los manifestantes con gases lacrimógenos y chorros de agua. Del mismo modo, se debe resaltar la respuesta por parte del gobierno hacia estos actores sociales, pues éste no solo amenazó a los estudiantes con la cancelación del semestre sino que recurrió a la ESMAD para reprimir las movilizaciones. Asimismo, tildó a los estudiantes de desconocer el contenido de la reforma. Por esto, los estudiantes participaron en debates públicos en el Congreso, la radio, universidades y la televisión, en los que refutaron, demostraron y argumentaron que conocían la ley 30 de 1992 y lo que planteaba la reforma.

\section{El sonado Paro Nacional}

Por otro lado, pero no menos importante, un último repertorio utilizado es el Paro Nacional. Para nosotras, este es el repertorio más significativo puesto que con éste los estudiantes demostraron su capacidad organizativa y cohesionadora. Según Liévano, "existían dos formas

1 CARACOL NOTICIAS (28 de Octubre de 2011) Policías que estuvieron en abrazatón piden se difundan fotografias.

2 Consignas de los estudiantes encontradas en el video que lleva por título No a la Ley 30 ni a su reforma - Carnaval Nocturno Univalle. 
de llegar al paro. La primera, era a través de Asambleas permanentes. Ellas implicaban que no habia clases y se reunían dos o tres veces por semana para discutir los repertorios de movilización [...] la segunda, por su parte consistía en paros escalonados, en los que la mitad del tiempo se veía clase y la otra mitad se discutía sobre el tema”.

La importancia del Paro Nacional, radica en que anteriormente se daban paros esporádicos que no tenían repercusiones nacionales. Sin embargo, en este caso el gobierno se enfrenta con que 32 universidades públicas entran en paro. Además, estas cuentan con el apoyo de muchos de las universidades privadas.

Sin embargo, los tres estudiante entrevistados, hacen énfasis en el problema que trajo consigo la discusión de entrar en paro y finalizar este, ya que no era fácil llegar a un consenso entre las diferentes universidades. A partir de esto, la MANE definió tres criterios para terminar el Paro: primero, que se retirara la Reforma a la ley; segundo, que se fortaleciera el movimiento estudiantil, y tercero, que se respetaran las movilizaciones sociales. Por lo tanto, se decide suspender el Paro no solo porque se cumplieron los tres criterios sino también porque no levantarlo los hacia ver como simples revoltosos y faltos de palabra. De igual manera, no se quería desgastar el modo de lucha como sucedió en Chile, por si tocaba volver a implementarlo.

En consecuencia, vemos que la MANE ha logrado el apoyo y legitimidad de la opinión pública, debido a estos nuevos repertorios que le permitieron transformar y romper con la concepción y los prejuicios que se tenían sobre los movimientos sociales. Pues es imposible olvidar como en nuestro país, cualquier persona que exprese una opinión contraria a la del gobierno o busque revindicar sus derechos, era y es tachada de guerrillero, terrorista, delincuente, entre otros apelativos. Un ejemplo claro de esto es lo que sucedió con la manifestación del Movimiento Político Marcha Patriótica en 2012, en la que no pasó mucho tiempo para que fueran señalados de tener vínculos con grupos ilegales.

\section{Se termina el Paro ¿y ahora qué?}

Una vez retirado el paro, el gobierno le exige al movimiento plantear una propuesta para la educación, en ese momento la MANE entra en su segunda dinámica y diseña un plan meto- 
dológico para construir una propuesta de ley de educación, completamente nueva que responda a las necesidades reales de la sociedad. Para ello, se conformaron mesas de discusión al interior de las universidades, después las propuestas de cada una de estas se desarrollaron en una mesa de articulación regional a finales de mayo del 2012.

En el caso del Valle del Cauca este encuentro regional se llevó a cabo en la Universidad del Valle. Pero, este encuentro regional no se llevó a cabo en todos los departamentos. Para el mes de junio, se realizó un encuentro programático llamado Primer Encuentro Social y Popular "Por una nueva educación para un país con Democracia, Soberanía y Paz" en el cual se articularon algunas de las conclusiones regionales, con el fin de "avanzar en la construcción del Documento de Exposición de Motivos” (MANE, 2012).

Los encargados de presentar dicha propuestas son los 25 voceros elegidos en marzo del 2012, quienes tienen la suficiente legitimidad para representar a todo el movimiento en las discusiones con la ministra, pues fueron elegidos democráticamente de los delegados de cada universidad, designados con anterioridad por las Asambleas estudiantiles $^{4}$. No obstante, la reunión con el gobierno nacional, no se realizó en el tiempo estipulado, puesto que no lograron consolidar una propuesta.

Una de las razones por las cuales no se logró llegar con una propuesta, tiene que ver con que no es lo mismo diseñar una nueva ley cuando se encuentran en Paro, que dedicarse al diseño de ésta y a responder a sus estudios al mismo tiempo. De igual manera, como indica Santiago Rojas "los estudiantes deben actuar de forma rápida y eficaz para que el Estado no tenga tanto tiempo para reaccionar e imponer sus leyes".

Finalmente, vemos que a pesar de las dificultades que ha tenido que enfrentar la MANE, los repertorios y las consignas de los estudiantes lograron tener un efecto directo en la opinión pública, alcanzando así legitimidad y alta aceptación por parte de la sociedad; y no una sociedad limitada a la academia, donde el estudiante tiene voz y voto, sino que también se amplían sus espacios de participación, porque logran influir en las políticas del gobierno y sus opiniones tienen peso en las decisiones que se tomen dentro del Congreso.

Sin embargo, no podemos prefijar el tiempo de permanencia del movimiento social, debido a que al enfrentarse a la segunda dinámica, han existido dificultades al interior del

4 Mayor información en el Observatorio de la Universidad Colombiana, http://www.universidad.edu.co/ index.php 
movimiento, ya que son un grupo compuesto por una sociedad heterogénea y que no cuenta con suficiente tiempo como para dedicarse a la creación de una nueva propuesta de ley. Esto se pone de manifiesto en los problemas que han tenido para cumplir con las fechas estipuladas.

\section{Bibliografía}

ARCHILA, Mauricio (2001). "Vida, pasión y ...de los movimientos sociales". En: Archila, Mauricio y Pardo, Mauricio (eds.) Movimientos sociales, Estado y democracia en Colombia. Colombia: Litocamargo Ltda., Universidad Nacional de Colombia.

GOHN, Maria (2002). "Procesos de construcción de ciudadanía global". En: Formación de lideres y movimientos sociales experiencias y propuestas educativas. Ediciones Abya-yala

RINCÓN, Andrés y Oscar Aponte (20 de enero de 2012). La movilización ha sido ese músculo que permitió avanzar al movimiento estudiantil. Consulta realizada desde: http://goo.gl/2682fN

VARGAS, Alejo (1996). "Hacia la gobernabilidad democrática en Colombia al final del siglo". En: Constitución, gobernabilidad y poder. Colombia: Universidad Nacional de Colombia.

\section{Documentos de prensa}

BEJARANO, Ramiro (13 de noviembre de 2011). "De estudiantes y fuero militar". En: El Espectador. Consulta realizada desde: http://www.elespectador.com/impreso/opinion/ columna-310944-de-estudiantes-y-fuero-militar

CARACOL NOTICIAS (28 de Octubre de 2011). Policias que estuvieron en abrazatón piden se difundan fotografias. Recuperado el 20 de julio de 2012, desde: http://www.mineducacion.gov.co/observatorio/1722/article-287995.html

EL COLOMBIANO (10 de noviembre de 2011). Presidente Santos reitera que retirará proyecto si cesa paro estudiantil. En: http://goo.gl/oh2oCU

- (11 de octubre de 2011). Marcha estudiantil en Medellín sale este miércoles del ITM. Consulta realizada desde: http://goo.gl/RzggCG (24 de noviembre de 2011). Estudiantes colombianos se unieron a marcha latinoamericana. Consulta realizada desde: http://goo.gl/0LIXle 
(25 de octubre de 2011). Cancelación del semestre académico depende de cada universidad: mineducación. Consulta realizada desde: http://goo.gl/wgL9RD - (31 de enero de 2012). Mane elegirá a sus voceros en marzo. Consulta realizada desde: http://www.elcolombiano.com/BancoConocimiento/M/mane_elegira_a_ sus_voceros_en_marzo/mane_elegira_a _sus_voceros_en_marzo.asp

EL ESPECTADOR (11 de octubre de 2011). Preparan paro estudiantil en Colombia. Consulta realizada desde: http://www.elespectador.com/noticias/educacion/video304865-preparan-paro-estudiantil-colombia

- (17 de octubre de 2011). Besatón por una educación pública, gratuita $y$ de calidad. Consulta realizada desde: http://www.elespectador.com/noticias/educacion/ articulo-305953-besaton-una-educacion-publica-gratuita-y-de-calidad

(9 de noviembre de 2011). La resurrección del movimiento estudiantil.

Consulta realizada desde: http://www.elespectador.com/impreso/vivir/articulo-310381-resurreccion-del-movimiento-estudiantil

EL NUEVO DÍA (29 de enero de 2012). Primer encuentro de la Mane. Consulta realizada desde: http://www.elnuevodia.com.co/nuevodia/especiales/sucesos/130390primer-encuentro-de-la-mane

EL TIEMPO (12 de marzo de 2012). Pedirán al Gobierno no presentar proyecto propio de reforma educativa. Consulta realizada desde: http://www.eltiempo.com/vida-de-hoy/ educacion/ARTICULO-WEB-NEW_NOTA_INTERIOR-11334581.html

(6 de abril de 2011). La marcha en contra de la reforma educativa, sin graves disturbios. Consulta realizada desde: http://www.eltiempo.com/vida-de-hoy/educacion/ARTICULO-WEB-NEW_NOTA_INTERIOR-9131911.html

FEU COLOMBIA (s.f.). Ponencia de la FEU para la MANE. Consulta realizada desde: http://feucolombia.org/index.php?option=com_content\&view=article\&id=66:ponenciama ne $\&$ catid $=41$ : ituacionuniversitaria $\&$ Itemid $=82$

MANE (30 de mayo de 2012). Primer Encuentro Social y Popular "Por una nueva educación para un país con Democracia, Soberanía y Paz". MANE Colombia - Bogotá 8-11 de Junio de 2012. Recuperado el 16 de marzo de 2013, de MANE Colombia. Consulta realizada desde: http://manecolombia.blogspot.com/2012/05/primerencuentro-social-y-popular-por.html 
(6 de abril de 2011). Conclusiones del ENEU. Marzo de 2011 - Mesa de Organización. Consulta realizada desde: http://funcomisionesmodep.org/index.php?option=com content\&view= category\&id $=50$ \&layout $=$ blog\&Itemid $=37 \&$ limitstart $=20$

OBSERVATORIO DE LA UNIVERSIDAD COLOMBIANA (12 de marzo de 2012). La MANE elige sus voceros, y devela divisiones entre los estudiantes. Consulta realizada desde: http://goo.gl/4yro4b

REVISTA SEMANA (12 de marzo de 2012). La MANE eligió a sus voceros. Consulta realizada desde: http://www.semana.com/nacion/mane-eligio-voceros/173633-3.aspx

TERRA (19 de octubre de 2011). Besatón de la Universidad Nacional estuvo a pedir de boca. Consulta realizada desde: http://goo.gl/pM2LTY

VANGUARDIA (21 de octubre de 2011). Con 'pupitrazo' se exigió retiro del proyecto de reforma a la educación. Consulta realizada desde: http://goo.gl/ULc1 AN

\section{Videografia}

ASUNTOS ESTUDIANTES. (05 octubre 2011). Educación gratuita para todos! No a la ley 30, no a la reforma neoliberal de Santos! [Archivo de Video]. Consulta realizada desde: http://www.youtube.com/watch?v=L8vjPSV_54

ABRIL TACITURNO (7 de noviembre de 2011). No a la Ley 30 ni a su reforma - Carnaval Nocturno Univalle [Archivo de Video]. Consulta realizada el 10 de octubre de 2012, desde: http://www.youtube.com/watch?v=UlCebsilf60\&feature=related

SUAREZ, León (2010, octubre 19). Consignas estudiantes UN [Archivo de Video]. Consulta realizada desde: http://www.youtube.com/watch?v=X_PVqHE8tZA

ZONA PÚBLICA (5 de abril de 2012). Primer debate público Universidad Sociedad [Archivo de Video]. Consulta realizada desde: http://www.youtube.com/watch?v=eke2C2e mDQo\&feature=player_embedded\#! 